\title{
Éditorial
}

\section{VII $^{\mathrm{e}}$ forum de biodétérioration des matériaux}

\footnotetext{
P
} armi les risques industriels nécessaires à maîtriser, les phénomènes de biodégradation représentent un danger réel, indispensable à caractériser. À ce jour, les mécanismes aboutissant à la dégradation des matériaux par la microflore bactérienne ne sont pas tous totalement explicités.

Il est largement reconnu que les micro-organismes favorisent la dégradation de la plupart des matériaux dans leur milieu naturel, y compris les matériaux métalliques. Dans ce dernier cas, la présence de colonies bactériennes sous forme de biofilm influence l'amorçage et la propagation de la plupart des attaques par corrosion communément rencontrées. En d'autres termes, la biocorrosion n'est pas une forme particulière de corrosion mais une modification des cinétiques sous l'influence de la présence des bactéries et des produits de leur métabolisme. Il est à noter que ce phénomène longtemps ignoré s'est imposé à la suite de recherches pour élucider certaines attaques de corrosion totalement inexplicables sans prendre en compte les modifications locales de l'interface métal/milieu provoquées par la présence des bactéries. De plus, l'expérience a montrél'omniprésence de la biocorrosion même dans des milieux très peu corrosifs et sur des métaux réputés résistants à la corrosion ou insensibles aux bactéries.

L'une des principales raisons de la lenteur des investigations scientifiques du domaine est la nature pluridisciplinaire du problème. En effet, la progression de la connaissance nécessite un dialogue soutenu entre métallurgistes, microbiologistes, biochimistes, chimistes et corrosionnistes. Une seconde raison, non moins négligeable, est le manque d'études scientifiques réalisées à partir de micro-organismes isolés de l'environnement industriel. On peut y ajouter le caractère parfois aléatoire ou transitoire de la corrosion bactérienne qui rend parfois très difficile l'expertise sur des matériaux extraits de leur environnement.

L'objet de ce numéro spécial est de faire le point sur les acquis disponibles et sur les récents développements réalisés par les équipes francophones travaillant sur ce sujet. Il contient les principales communications présentées lors du $7^{\mathrm{e}}$ forum de biodétérioration des matériaux organisé à Caen les 26 et 27 mai 2005 par la Commission Biodétérioration des Matériaux du CEFRACOR (CEntre FRançais de l'AntiCORrosion), avec le parrainage de la SFM (Société Française de Microbiologie). Les différents travaux qui y ont été présentés illustrent largement l'interdisciplinarité du domaine. Les investigations portant sur les principaux matériaux (métaux, bétons, pierres) utilisés par l'industrie ont été présentées. Les moyens mis en œuvre dans les différentes études sont variés : mesures électrochimiques (courbes de polarisation, impédance), analyses chimiques (IRTF, ICP-AES...), analyses microbiologiques (technique NPP) ou observations microscopiques (MEB). On trouvera donc ici, dans une première partie, les articles illustrant les conférences plénières avec notamment P.G. Rouxhet qui décrit les concepts de D. Haras essentiels pour comprendre le comportement des enzymes et des microorganismes aux interfaces. Une revue des principaux phénomènes d'altération liés aux biofilms ainsi que des stratégies de prévention. Enfin une synthèse bibliographique, présentee par D. Féron, illustre les principaux résultats connus de la littérature concernant les situations les plus défavorables à la résistance à la corrosion localisée des aciers inoxydables en eau de mer naturelle. L'article porte notamment sur l'influence du couplage des conditions aérobies et anaérobies, associé à la présence de sulfures qui diminue la résistance de la couche passive. Dans une seconde partie, J. Landoulsi décrit les hypothèses enzymatiques susceptibles d'expliquer les mécanismes impliqués dans le comportement électrochimique des aciers inoxydables dans les eaux naturelles. Les résultats d'E. Dajoux soulignent le rôle joué par le chrome sur le comportement des bactéries marines et la corrosion généralisée en eau de mer naturelle. La confirmation ou l'infirmation du phénomène d'accroissement du potentiel libre de corrosion d'un acier inoxydable plongé dans l'eau douce naturelle est également discuté par C. Marconnet. L'article de R. Sabot met l'accent sur l'effet des différentes substances organiques vis-à-vis des processus de corrosion des aciers, en particulier des lactates qui modifient le produit de l'oxydation de la rouille verte sulfatée. Des travaux portant sur les problèmes d'inhibition de la corrosion sont également présentés : ainsi, F. Bach démontre l'effet des microorganismes sur la baisse de rendement d'un inhibiteur organo-minéral susceptible de préserver les maçonneries anciennes. La résistance des matériaux cimentaires est discutée dans les travaux de A. Bertron et S. Roux. Ces travaux soulignent l'importance du facteur biologique 
dans la dégradation des bétons en contact avec de l'eau douce naturelle ou des effluents agricoles. Finalement, deux articles décrivent des résultats très encourageants à court terme puisqu'ils démontrent d'une part le rôle bénéfique de l'oxyde d'étain pour la protection contre les salissures des dispositifs optiques immergés en milieu marin (S. Haskouri), et d'autre part, l'intérêt de la technique d'analyse par spectroscopie infrarouge à transformée de Fourier pour l'identification rapide de la flore sulfurogène impliquée dans les phénomènes de biodétérioration (N. Boudaud).

Thierry Braisaz

Laboratoire CORRODYS

Pour le comité d'organisation
Gérard Pinard Legry Président du CEFRACOR 